\title{
Detecting and Analyzing System for the Vibration Comfort of Car Seats Based on LabVIEW
}

\author{
Ying Qiu \\ Key Laboratory of Conveyance and Equipment, Ministry of Education \\ School of Mechanical and Electronical Engineering, East China Jiaotong University, \\ Nanchang, P.R. China \\ Qiuying72@163.com
}

\begin{abstract}
In this research, LabVIEW 8.5 is taken as the software platform. Sensors, data acquisition cards are combined as its hardware. A detecting and analyzing system for the vibration comfort of car seats is developed, in which the functions as to the collecting and processing of vibrating signals, the access of data and the display of images are achieved. The system can also analyze the dynamic characteristics and the comfort of the occupant-seat system. By choosing different seat characteristics according to different vehicle environments, the best comfort can be obtained. In addition, it also provides some favorable references to the designing of safe and comfortable automobile seats.
\end{abstract}

Keywords: Vehicle seat, LABVIEW, Dynamic characteristics, Vibration detection.

\section{Introduction}

Car seat is an important part of the car. Its main function is to support the driver and the passenger's body, to reduce the impact on the body aroused by rough road surface and to attenuate the resulting vibrations. With the improvement of living standard and the rapid development of automobile technology, the demand for automotive comfort is higher and higher. Automotive comfort includes static and dynamic comfort. The former mainly involves size parameters, surface quality and the regulation characteristics; and the latter is largely related to vibration characteristics. In mechanical vibration test, because numerous complex test equipment are needed, a simple test may even require great manpower and large quantity of material resources, not to mention some complex work like the vibration test on car seats. With the development of computer and software technology, it is taking over the traditional method and is becoming the trend in the area of testing. In this article, a development platform based on LabVIEW software (a virtual instrument platform initiated by American National Instruments Company) is constructed. Necessary sensor, signal conditioners and data acquisition cards are taken as the main hardware to build a detecting and analyzing system for the seat vibration test. Results from experiments show that the suggested 
system can effectively fulfill the work of acquiring and processing seat vibration signals, analyzing its dynamic characteristics and assessing its comfort rate.

\section{Overall System Structure and Working Principle}

Using virtual test instruments, the Virtual vibration testing and analyzing system completes the work of measuring, data analysis and processing of mechanical vibration signals. Totally resorting to computer software, it successfully achieves the functions of vibration signal acquisition, display, access and processing. The system structure is illustrated in Figure1.

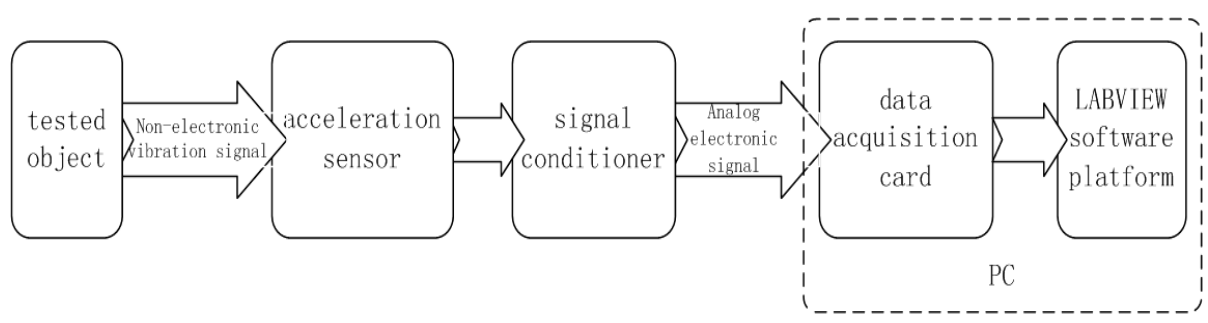

Fig. 1. Schematic diagram of system structure

Hardware for the system includes: acceleration sensor, signal amplifier suitable for transfer, data acquisition card and PC machines. Its software is realized through graphic-oriented software LabVIEW.

The seat vibration test and analysis system first transforms the vibration signals detected by the sensor into analog signals, and then passed it to the signal conditioning. Next, the analog electronic signal is converted into digital signal by data acquisition card and finally is passed to the vibration test and analysis computer software for analysis.

The system realizes the seat vibration signal testing and data analysis via computer, which can completely replace the conventional signal analyzer and many other hardware devices to complete a variety of signal analysis and processing.

\section{Designing of System Functions}

Based on the needs of seat vibration comfort testing, the main functions of this system include vibration signal acquisition and display, signal analysis and processing, dynamic characteristics analysis and comfort-rating assessment on seat vibration. Block diagram of the system function is shown in Figure 2. 


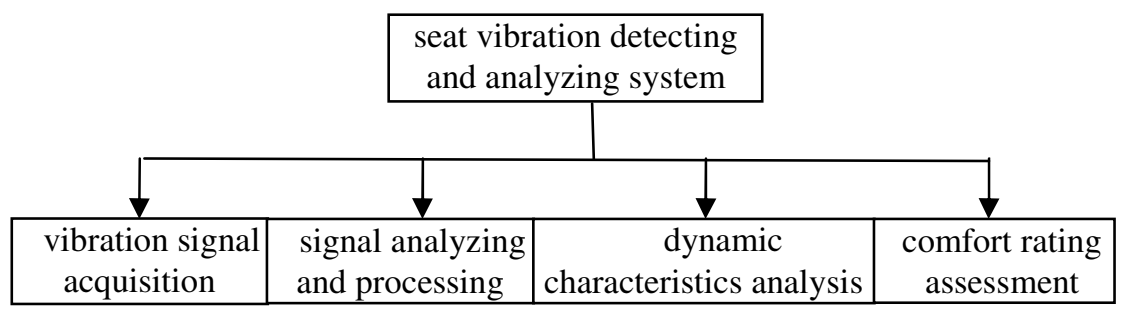

Fig. 2. Block diagram of the system function

\section{Implementation and Testing of System Functions}

Front panel of the seat vibration test system is shown in Figure 3, the functional modules can be switched freely through the option cards to achieve the functions of signal acquisition, signal processing, dynamic analysis and evaluation of comfort.

\subsection{Vibration Signal Acquisition Module}

The system completes the external circuit signal acquisition through the DAQ assistant acquisition module and NI ELVIS external board. The acquisition results are shown in Figure 3.

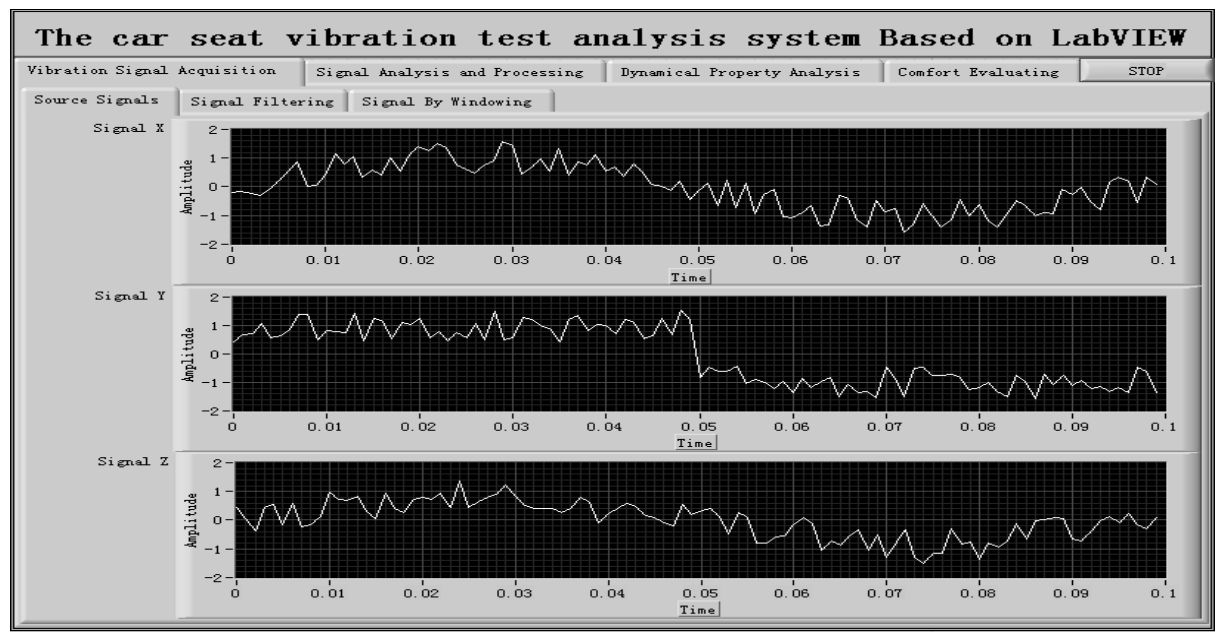

Fig. 3. Front panel of the seat vibration test system

\subsection{Signal Analysis and Processing Module}

In order to eliminate the interference aroused by external signals on the vibration signals, the acquired signals must be pretreated. The system mainly makes some 
filtering and spectrum analysis on analog signals mixed with noise, in order to complete the module test. The mathematics algorithm for filtering is rather complicated, but LabVIEW has modularized the filtering function, so it is quite easy to complete the filtering program with these functions. Signals mixed with noise have been filtered and a comparison between the source signal and the filtered signals will clearly demonstrate the effect as shown in Figure 4.

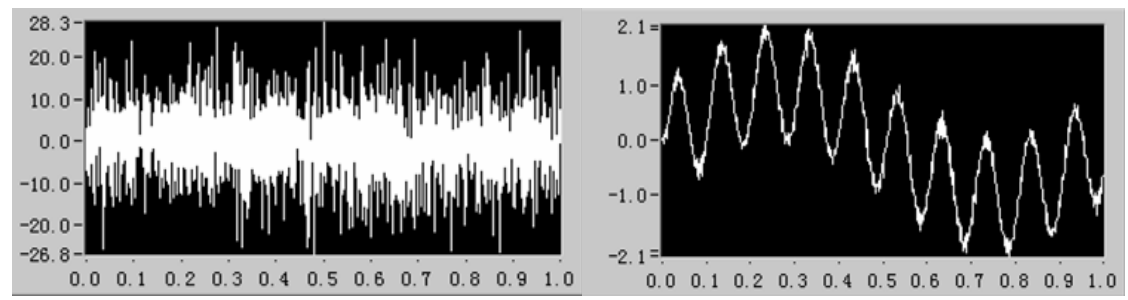

Fig. 4. Effect of filtering

Besides filtering the signals, the module also has many other functions including frequency domain analysis, window processing, correlation analysis, FFT transform and etc.

\subsection{Dynamics Analysis Module}

The main function of car seats is to support the driver and the passenger's body, to reduce the impact of uneven road surface and to attenuate the resulting vibrations passed to the people, so as to provide a comfortable and safe riding condition. The kinetic parameters --- natural frequency and the damping ratio of the car seat, have a major impact on the damping properties of the seat. Thus, take a representative 1-DOF and 3-DOF occupant-seat model for dynamic analysis. Suggestions on how to choose dynamic parameters are also presented. The structure of 1-DOF and 3-DOF occupant-seat model is shown in figure 5.

In 1-DOF model, the human body is considered as a rigid body on the seat. Its frequency response function is:

$$
|H(\omega)|=\sqrt{\frac{1+(2 \xi \gamma)^{2}}{\left(1-\gamma^{2}\right)^{2}+(2 \xi \gamma)^{2}}}
$$

Where: $\xi=C /(2 \sqrt{K M})$ is the damping ratio, $\gamma=\omega / \sqrt{K / M}$ is the frequency ratio.

The seat damping ratio and frequency ratio can be adjusted by changing the parameters of the car seat, so as to improve the frequency response of the seat. 

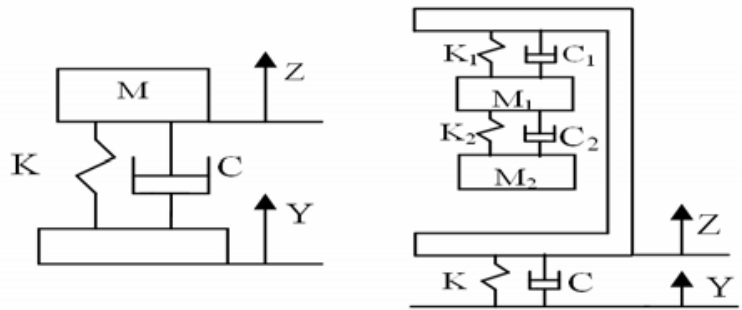

Fig. 5. Structure of 1-DOF and 3-DOF occupant-seat model

In 3-DOF model, the human body is considered as a 2-DOF dynamic model on the seat. Suppose: $\omega_{n}=\sqrt{K /\left(M_{1}+M_{2}\right)}, \omega_{n 1}=\sqrt{K_{1} / M_{1}}, \omega_{n 2}=\sqrt{K_{2} / M_{2}}, \gamma=\omega / \omega_{n}$, $\gamma_{1}=\omega / \omega_{n 1} \quad, \quad \gamma_{2}=\omega / \omega_{n 2} \quad, \quad \xi=C /\left(2 \sqrt{K\left(M_{1}+M_{2}\right)}\right) \quad, \quad \xi_{1}=C_{1} /\left(2 \sqrt{K_{1} M_{1}}\right) \quad$, $\xi_{2}=C_{2} /\left(2 \sqrt{K_{2} M_{2}}\right), \mu_{1}=M_{1} /\left(M_{1}+M_{2}\right), \mu_{2}=M_{2} /\left(M_{1}+M_{2}\right)$, The frequency response function is:

$$
H(\omega)=\frac{1+j 2 \xi \gamma}{(1+j 2 \xi \gamma)-\left(\mu_{1} B_{1}+\mu_{2} B_{2}\right)}
$$

Where: $B_{1}=\frac{1+j 2 \xi_{1} \gamma_{1}}{1-\gamma_{1}^{2}+j 2 \xi_{1} \gamma_{1}}, \quad B_{2}=\frac{1+j 2 \xi_{2} \gamma_{2}}{1-\gamma_{2}^{2}+j 2 \xi_{2} \gamma_{2}}$.

When analyzing the simulation in LabVIEW, the parameters in the model are set on the following basis: 1) "Test Method of Car Seat Dynamic Comfort", in which the standard is $\mathrm{M}=51 \mathrm{~kg}$; 2) statistic results from domestic human body vibration test, in which M1 $=29.8 \mathrm{~kg}, \mathrm{M} 2=5.5 \mathrm{~kg}, \mathrm{~K} 1=224.8 \mathrm{~N} / \mathrm{cm}, \mathrm{K} 2=133.1 \mathrm{~N} / \mathrm{cm}, \mathrm{C} 1=3.9 \mathrm{~N} /$ (cm.s), $\mathrm{C} 1=1.9 \mathrm{~N} /(\mathrm{cm} . \mathrm{s})$. The frequency response function of the two models is related not only to the parameters of the human body model but also to the natural frequency and damping ratio of the seat.

Figure 6 shows the system frequency response curve under different natural frequency and damping ratio of 1-DOF model, by which reasonable parameters can be chosen.
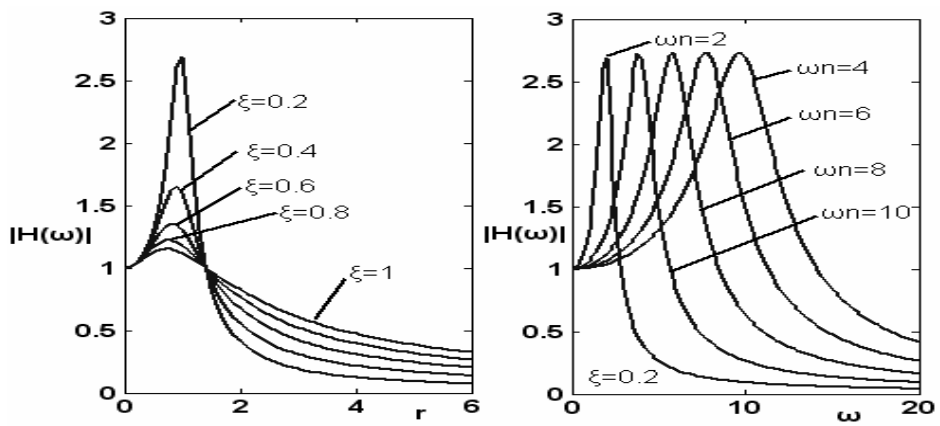

Fig. 6. Frequency response curve under different damping ratio and natural frequency 
The module can also analyze the displacement response curve of human body vibration by simulating different car environment and road condition. When different seat parameters are selected, the changes of human body vibration displacement can be directly observed, which will provide favorable reference for the design of safe and comfortable seats.

\subsection{Seat Comfort Assessment Module}

The function of data analysis module is to analyze the collected and pretreated signals, so that the specific values of comfort can be obtained and car seat comfort can be measured. Data is processed using total RMS value of weighted acceleration comparison method by the data analysis module.

Taking into account individual's different sensitivity to the frequency of vibration, this method converts RMS acceleration which frequency ranges within $1 \sim 80 \mathrm{~Hz}$ into RMS acceleration within sensitive frequency $(4 \sim 8 \mathrm{~Hz}$, or $1 \sim 2 \mathrm{~Hz}$ ) by multiplying a different frequency weighting, then calculates the total acceleration RMS, and then sets the standard for evaluation: whether the corresponding limit exceeds ISO2631 sensitivity limit of the frequency or length of time.

As for the composite vibration condition which human body withstand from $\mathrm{X}, \mathrm{Y}$, $\mathrm{Z}$ three directions, we can first obtain respective frequency weighted RMS by calculating the acceleration spectra in each vibration direction, and then calculate the combined weighted acceleration value $\mathrm{A}_{\omega}$.

$$
A_{\omega}=\sqrt{\left(1.4 a_{x \omega}\right)^{2}+\left(1.4 a_{y \omega}\right)^{2}+\left(a_{z \omega}\right)^{2}}
$$

Where: the factor of 1.4 is the most sensitive in the body within the same frequency range corresponding vertical and horizontal and vertical curve ratio.

"Total Travel Value Method" has been introduced in the draft of ISO2631/CD1991, and the relationship between the acceleration value and the subjective feeling of the occupant has been given, as is shown in Table 1.

Table 1. Relationship between Acceleration Value and Subjective Feeling of the Occupant

\begin{tabular}{|c|c|}
\hline Weighted Acceleration Value $\left(\mathrm{m} / \mathrm{s}^{2}\right)$ & Subjective Feeling of the Occupant \\
\hline$<0.0315$ & Comfortable \\
\hline $0.315 \sim 0.63$ & slightly uncomfortable \\
\hline $0.5 \sim 1.0$ & somewhat uncomfortable \\
\hline $0.8 \sim 1.6$ & Uncomfortable \\
\hline $1.25 \sim 2.5$ & very uncomfortable \\
\hline$>2.0$ & Unbearably Uncomfortable \\
\hline
\end{tabular}

The vibration signals from $\mathrm{X}, \mathrm{Y}$ and $\mathrm{Z}$ direction are measured by the sensor. Then they are converted into vehicle vibration comfort indicators $C_{\omega}$ and combined weighted RMS $A_{\omega}$ through the program operation. Finally, the comfort condition is displayed on the front panel. 


\section{Conclusion}

This system is actualized through a powerful graphical programming language LabVIEW and the existing hardware devices. It can measure seat vibration, process data, analyze seat dynamics characteristic and evaluate the driving comfort. Compared with traditional method, this system has the following advantages:

(1) Computer software program has replaced the traditional spectrum analyzers and other hardware devices. This system validates real-time analysis and processing, reduces test costs and shortens the test cycle.

(2) Resorting to the existing signal processing modules of LabVIEW to deal with vibration signals, the process is quicker and more accurate, and thus complex mathematic calculation is avoided.

(3) By making a dynamic simulation analysis on the human body - seat model, it provides a favorable reference to the designing of safe and comfortable seats. Based on ISO2631 standard, seat driving comfort is also evaluated.

Acknowledgement. This work is supported by the Research Foundation of ECJTU (NO. 01308115), and Key Laboratory of Conveyance and Equipment.

\section{References}

1. Boileau, P. E.: A body Mass Dependent Mechanical Impendence Model for Application in vibration Seat Testing. J. Journal of Sound and Vibration 253(1), 243-264 (2002)

2. National Instruments Corp LabVIEW User Manual, Austin, Texas, USA, pp. 27-444 (1998)

3. National Instruments Corp BridgeVIEW and LabVIEW G Programming Reference Manual, Austin, Texas USA, pp. 27-566 (1998)

4. Dai, X., Sun, H., Ou, J.J.: Virtual instrument design for mechanical vibration measurement. In Chinese. China Measurement \& Testing Technology 34(4), 92-95 (2008)

5. Meng, Y.M., Gao, F.W., Duan, J.L., Li, S.P., Huang, B.P.: The research of vibration measurement and analysis system based on LABVIEW. in Chinese. Journal of Guangxi University (Nat. Sci. Ed.) 32(2), 114-117 (2007)

6. Qian, Y., Zhou, Y.P., Chen, J.W.: Analytical System of Vibration Measurement Based on LabVIEW. In Chinese. Journal of Wenzhou University, 58-61 (2005)

7. Chen, X.W., Liu, Y.: Realization of test analysis for vibration signal based on LabVIEW. In Chinese. Electronic Measurement Technology, 108-120 (2008) 